\title{
Zero-dimensional Gorenstein Algebras with the Action of the Symmetric Group
}

\author{
Hideaki Morita(*) - Akinito Wachi(**) - Junzo Watanabe(***)
}

\section{Introduction.}

Suppose that $A$ is a Gorenstein $K$-algebra with a strong Lefschetz element $l \in A$. Put $L=\times l \in \operatorname{End}(A)$. Then it is possible to construct a degree -1 map $D \in \operatorname{End}(A)$ such that $\{L, D, H\}$ is an $\mathfrak{s}(2)$-triple, $H=[L, D]$, and the weight space decomposition coincides with the natural grading decomposition. This means that the eigenspaces of $H$ are precisely the homogeneous parts of $A$. Suppose moreover that the symmetric group $S_{k}$ acts on $A$ as permutation of the variables and that the invariant linear form $l=x_{1}+x_{2}+\cdots+x_{n}$ is a strong Lefschetz element. Then, as is obvious, the vector space $\operatorname{Ker} L$ is fixed, hence the spaces $\operatorname{Ker} L \cap A_{i}$ are fixed by the action of $S_{k}$. Thus in such a case an irreducible decomposition of $A$ can be constructed by first decomposing the kernel of the multiplication map

$$
\times\left(x_{1}+\cdots+x_{k}\right): A \rightarrow A
$$

into irreducible spaces and then applying the map $D$ repeatedly to the constituents of the decomposition of $\operatorname{Ker} L$. (Or equivalently, first decompose $\operatorname{Ker} D$ and then apply $L$.)

An obvious example of an Artinian Gorenstein algebra in which $l=x_{1}+\cdots+x_{k}$ is a strong Lefschetz element is the equi-degree mono-

(*) Indirizzo dell'A.: Muroran Institute of Technology, Muroran 050-8585, Japan.

E-mail: morita@muroran-it.ac.jp

(**) Indirizzo dell'A.: Division of Comprehensive Education, Hokkaido Institute of Technology, Sapporo 006-8585, Japan.

E-mail: wachi@hit.ac.jp

(***) Indirizzo dell'A.: Department of Mathematics, Tokai University, Hiratsuka 259-1292, Japan.

E-mail: watanabe@sm.u-tokai.ac.jp 
mial complete intersection

$$
A(n, k)=K\left[x_{1}, \cdots, x_{k}\right] /\left(x_{1}^{n}, \cdots, x_{k}^{n}\right) .
$$

It is possible to identify $A(n, k)$ with the tensor space

$$
\left(K^{n}\right)^{\otimes k}
$$

as $\left(G L(n) \times S_{k}\right)$-modules. The purpose of this paper is to understand the strong Lefschetz property of $A(n, k)$ with $l$ a strong Lefschetz element in the context of the Schur-Weyl duality.

Let $Y^{T}$ be the Young symmetrizer in $K\left[S_{k}\right]$ corresponding to a Young tableau $T$. One might ask first what is the Hilbert series of

$$
S_{k} \cdot Y^{T}(A(n, k))
$$

the maximal isotypic submodule of $A(n, k)$ corresponding to the shape of $T$. It turns out that it equals the $q$-analog of the Weyl dimension formula. Since it does not seem to be well known and since we have been unable to find a good reference, we give a proof for it in section 6 . This is stated in Proposition 27.

Proposition 27 is not a consequence of the strong Lefschetz property of $A(n, k)$, but Corollary 36, which states that the $q$-analog of the Weyl dimension formula is unimodal and symmetric, may be thought of as a consequence of the fact $l$ is a strong Lefschetz element.

Terasoma-Yamada [8] considered the coinvariant algebra of $S_{k}$,

$$
A=K\left[x_{1}, \cdots, x_{k}\right] /\left(e_{1}, \cdots, e_{k}\right),
$$

where $e_{i}$ is the elementary symmetric polynomial of degree $i$. Note that this is also a Gorenstein algebra with the action of $S_{k}$. They remark that the Hilbert series of $S_{k} \cdot Y^{T}(A)$ is obtained as the $q$-analog of the hook-length formula. Thus our situation is similar to theirs, but it is not true that the Hilbert series of $S_{k} \cdot Y^{T}(A)$ is unimodal and symmetric. The algebra $A$ above has the strong Lefschetz property, but no symmetric linear form is a strong Lefschetz element, which causes the difference.

In section 7 , we consider the irreducible representation

$$
\rho^{\lambda}: G L(n) \rightarrow G L\left(W^{\lambda}\right)
$$

of the general linear group $G L(n)$ on a finite dimensional vector space $W^{\lambda}$ and show how the Jordan matrix with a single eigenvalue $a$,

$$
J(a, n) \in G L(n)
$$

is transformed by $\rho^{\lambda}$. This is done again by identifying the Artinian algebra 
$A(n, k)$ with the tensor space $\left(K^{n}\right)^{\otimes k}$ as $\left(G L(n) \times S_{k}\right)$-modules so that the Schur-Weyl duality applies to $A(n, k)$. Here the consideration of the behavior of $l$ as a multiplication operator is indispensable. The two extremal cases of $A(n, k)$ treated in Sections 3 and 4 should be regarded as examples which show that our method is a new way to understand some classical results.

One other result of this paper is Theorem 22 in Section 5. We apply Theorem 19 to determine the minimal number of generators of the ideal

$$
\left(x_{1}^{2}, \cdots, x_{k}^{2}\right):\left(x_{1}+\cdots+x_{k}\right)
$$

in the polynomial ring. By analyzing Specht polynomials involving only square-free monomials, it is possible to determine a minimal generating set of the ideal.

The authors thank the referee very much for helpful comments and suggestions.

\section{Preliminaries.}

2.1 - A list of notational conventions.

Here is a list of notation which we are going to fix throughout the paper. Details follow the list.

- $K$ denotes an algebraically closed field of characteristic 0 .

- $\lambda=\left(k_{1}, k_{2}, \cdots, k_{r}\right) \vdash k$ indicates that $\lambda$ is a partition of a positive integer $k$. The same notation is used to indicate a Young diagram of size $k$. It is assumed that $k_{1} \geq k_{2} \geq \cdots \geq k_{r}>0$. The length $r$ of $\lambda$ is denoted by $l(\lambda)$.

- If $J \in \operatorname{End}(V)$ is nilpotent, we call the partition $\lambda(J)=\left(k_{1}, \cdots, k_{r}\right)$ the conjugacy class of $J$, indicating the sizes of Jordan blocks in the Jordan decomposition of $J$.

- A Young tableau $T$ is a Young diagram $\lambda$ with a numbering of boxes with integers $1,2, \cdots, k$. In this case $\lambda$ is the shape of $T$.

- $Y^{T}$ denotes the Young symmetrizer defined by the Young tableau $T$. It is an element of the group algebra $K\left[S_{k}\right]$.

- Suppose that $T$ is a Young tableau and $\lambda$ is the shape of $T$. If $V$ is an $S_{k}$-module, then $Y^{\lambda}(V)$ denotes $S_{k} \cdot Y^{T}(V)$. Thus $Y^{\lambda}(V)$ is the maximal submodule of $V$ consisting of modules isomorphic to the Specht module $V^{\lambda}$. We may regard $Y^{\lambda}($ ) as a functor to extract the isotypic component belonging to $\lambda$.

- $\Delta_{T}$ denotes the Specht polynomial defined by a Young tableau $T$. 
2.2 - The equi-degree monomial complete intersection as a tensor space.

Let $R=K\left[x_{1}, \cdots, x_{k}\right]$ be the polynomial ring. The partial degree of a homogeneous polynomial $f \in R$ is the maximum degree of $f$ with respect to a single variable. By $A(n, k)$ we denote the vector subspace of $R$ consisting of polynomials of partial degree at most $n-1$. Let $I$ be the ideal $I=\left(x_{1}^{n}, \cdots, x_{k}^{n}\right)$ of $R$. Then as vector spaces we have the decomposition

$$
R=A(n, k) \oplus I \text {. }
$$

The vector space $A(n, k)$ may be regarded as the tensor space $\left(K^{n}\right)^{\otimes k}$. Since $A(n, k) \cong R / I$ the vector space $A(n, k)$ has the structure of a commutative ring. Put $A=A(n, k)$. A basis of $A$ can be the set of monomials of partial degree at most $(n-1)$ :

$$
\left\{x_{1}^{i_{1}} \cdots x_{k}^{i_{k}} \mid 0 \leq i_{1}, \cdots, i_{k} \leq n-1\right\}
$$

An element of $A$ is expressed uniquely as

$$
\sum F\left(i_{1}, i_{2}, \cdots, i_{k}\right) x_{1}^{i_{1}} x_{2}^{i_{2}} \cdots x_{k}^{i_{k}} .
$$

With the identification $A \cong\left(K^{n}\right)^{\otimes k}$ the general linear group $G L(n)$ acts on the vector space $A$ as the tensor representation. Let

$$
\Phi: G L(n) \rightarrow G L(A)
$$

be the representation. Explicitly, if $g=\left(g_{\alpha \beta}\right) \in G L(n)$ then

$$
\Phi(g)\left(x_{1}^{j_{1}} \cdots x_{k}^{j_{k}}\right)=\left(\sum_{\alpha=0}^{n-1} g_{\alpha j_{1}} x_{1}^{\alpha}\right) \cdots\left(\sum_{\alpha=0}^{n-1} g_{\alpha j_{k}} x_{k}^{\alpha}\right) .
$$

Here the indices $\alpha, \beta$ of the matrix entries for $g=\left(g_{\alpha \beta}\right) \in G L(n)$ range over $0,1, \cdots, n-1$. At the same time the symmetric group $S_{k}$ acts on $A$ as the permutation of the variables.

We are interested in the decomposition of $A$ into irreducible $S_{k}$-modules. According to the Schur-Weyl duality it will give us an irreducible decomposition of $A$ as $\left(G L(n) \times S_{k}\right)$-modules.

\section{3 - Young tableaux and Specht polynomials.}

A partition of a positive integer $k$ is a way to express $k$ as a sum of positive integers. If we say that $\lambda=\left(k_{1}, \cdots, k_{r}\right)$ is a partition of $k$, it means 
that $k=k_{1}+\cdots+k_{r}$ and $k_{1} \geq \cdots \geq k_{r}>0$. A partition of $k$ is identified with a Young diagram of size $k$ in a well known manner. Thus the same notation $\lambda=\left(k_{1}, \cdots, k_{r}\right)$ denotes a Young diagram of size $\sum k_{i}$ with rows of $k_{i}$ boxes, $i=1, \cdots, r$, aligned left. A Young tableau $T$ is a Young diagram $\lambda$ whose boxes are numbered with integers $1, \cdots, k$ in any order. In this case we say that $\lambda$ is the shape of $T$. A Young tableau is standard if every row and column is numbered increasingly.

A Young tableau $T$ defines a Specht polynomial, denoted $\Delta_{T}$, as follows: Put $I=\{1,2, \cdots, k\}$ and

$$
I_{j}=\{\alpha \in I \mid \alpha \text { is in the } j \text { th column of } T\} .
$$

Define

$$
\Delta_{j}=\prod_{\substack{\alpha<\beta \\ \alpha, \beta \in I_{j}}}\left(x_{\alpha}-x_{\beta}\right)
$$

and finally,

$$
\Delta_{T}=\prod_{j} \Delta_{j},
$$

where $j$ runs over all columns. $\Delta_{T}$ is the Specht polynomial defined by the Young tableau $T$.

\section{4 - Nilpotent matrices and Jordan bases.}

Let $\mathbf{M}(k)$ denote the set of $k \times k$ matrices with entries in $K$. Let $J \in \mathbf{M}(k)$ be a nilpotent matrix. Let

$$
v_{i}=\operatorname{rank} J^{i}-\operatorname{rank} J^{i+1} \text { for } i=0,1, \cdots, p,
$$

where $p$ is the least integer such that $J^{p+1}=O$. Then $\widehat{\lambda}:=\left(v_{0}, v_{1}, \cdots, v_{p}\right)$ is a partition of $k$. We denote the dual partition of $\hat{\lambda}$ by $\lambda(J)$. (cf. Definition 1 below.)

Now suppose that $T$ is a Young tableau of size $k$. Using the numbering of $T$ define the matrix $J=\left(a_{i j}\right) \in \mathbf{M}(k)$ by

$$
a_{i j}= \begin{cases}1 & \text { if } j \text { is next to and on the right of } i \text { in } T, \\ 0 & \text { otherwise. }\end{cases}
$$

It is easy to see that the matrix $J$ is nilpotent and $\lambda(J)$ is the shape of $T$. We call any matrix defined as above for a Young tableau $T$ a Jordan canonical form (of a nilpotent matrix). 
Let $V$ be a vector space of dimension $k$. If a basis of $V$ is fixed, we may identify $\mathbf{M}(k)$ and $\operatorname{End}(V)$. Suppose that $J \in \operatorname{End}(V)$ is nilpotent. A Jordan basis for $J$ is a basis of $V$ on which $J$ is in a Jordan canonical form. (According to the definition of a Jordan basis just defined above, any permutation of basis elements of a Jordan basis is a Jordan basis.) Since $K$ is algebraically closed, there exists a Jordan basis. Note that if two nilpotent elements $J$ and $J^{\prime}$ are conjugate, then $\lambda(J)=\lambda\left(J^{\prime}\right)$. We make a definition of the "conjugacy class" of a nilpotent endomorphism, with a slight abuse of language, as follows.

Definition 1 . Let $V$ be a $k$-dimensional vector space over $K$. Suppose that $J \in \operatorname{End}(V)$ is nilpotent. We say that a partition $\left(k_{1}, \cdots, k_{r}\right)$ of $k$ is the conjugacy class of $J$ if the Jordan canonical form of $J$ consists of the Jordan blocks of sizes $k_{1}, \cdots, k_{r}$. We denote by $\lambda(J)$ the conjugacy class of $J$.

REMARK 2. Note that the notation $\lambda(J)$ coincides with the previously defined $\lambda(J)$ for a nilpotent matrix. In fact, if we put $v_{i}=\operatorname{dim} \operatorname{Im} J^{i} / \operatorname{Im} J^{i+1}$, then the sequence $\hat{\lambda}=\left(v_{0}, v_{1}, \cdots, v_{p}\right)$ is a partition of $k$. One sees easily that the dual partition $\lambda$ to $\widehat{\lambda}$ is the conjugacy class of $J$.

Let $J \in \operatorname{End}(V)$ be nilpotent with the conjugacy class $\lambda=\lambda(J)$. Suppose that $B \subset V$ is a Jordan basis for $J$. Then it is possible to place the elements of $B$ into the boxes of the Young diagram $\lambda(J)$ bijectively in such a way that it satisfies the following conditions:

$$
\left\{\begin{array}{l}
e, e^{\prime} \in B \text { and } e^{\prime}=J e \Leftrightarrow e^{\prime} \text { is next to and on the right of } e . \\
e \in \operatorname{Ker} J \Leftrightarrow e \text { is at the end of a row. }
\end{array}\right.
$$

(cf. Equation (6).)

REMARK 3. As explained above, by choosing a bijection between the elements of a Jordan basis for $J$ and the boxes of the Young diagram $\lambda(J)$, it is possible to identify a Jordan basis $B$ for $J$ and the Young diagram $\lambda(J)$. With this identification the rightmost boxes of $\lambda(J)$ form a basis for Ker $J$. Also the boxes of the first column of $\lambda(J)$ coincide with $\{b \in B \mid b \notin \operatorname{Im} J\}$. Once $\lambda(J)$ is known, $\operatorname{Ker} J \cap B$ determines $B$. Similarly the diagram $\lambda(J)$ and the subset $B \backslash \operatorname{Im} J \subset B$ determine $B$. 
2.5 - The strong Lefschetz property.

Let $V=\bigoplus_{i=0}^{c} V_{i}$ be a finite dimensional graded vector space. The Hilbert series of $V$ is the map $i \mapsto \operatorname{dim} V_{i}$, which we usually write as the polynomial $\sum\left(\operatorname{dim} V_{i}\right) q^{i}$. (For convention we let $\operatorname{dim} V_{i}=0$ for $i<0$ or $i>c$.) Let $J \in \operatorname{End}(V)$ be a map of degree one so $J$ consists of the graded pieces $\left.J\right|_{V_{i}}: V_{i} \rightarrow V_{i+1}$. Then $J$ is nilpotent. We say that $J \in \operatorname{End}(V)$ is a strong Lefschetz element if the restricted map

$$
\left.J^{c-2 i}\right|_{V_{i}}: V_{i} \rightarrow V_{c-i}
$$

is bijective for all $i=0,1, \cdots,[c / 2]$. (Such an endomorphism exists only if the Hilbert series of $V$ is symmetric and unimodal.)

Definition 4. Let $A=\bigoplus_{i=0}^{c} A_{i}$ be an Artinian graded $K$-algebra. Denote by $\times: A \rightarrow \operatorname{End}(A)$ the regular representation of $A$. (I.e., $\times a(b)=a b$ for $a, b \in A$.) We say that $A$ has the strong Lefschetz property, if there exists a linear form $l \in A$ such that $\times l \in \operatorname{End}(A)$ is a strong Lefschetz element. We call such a linear form $l \in A$ a strong Lefschetz element of $A$ as well as $\times l \in \operatorname{End}(A)$.

Proposition 5. Suppose that $A=\bigoplus_{i=0}^{c} A_{i}$ is a graded Artinian K-algebra with a symmetric Hilbert series $\sum h_{i} q^{i}$. Let l be a linear form of $A$. Then $\times l$ is a strong Lefschetz element if and only if $\lambda(\times l)$ is the dual partition to $\left(h_{0}^{\prime}, h_{1}^{\prime}, \cdots, h_{c}^{\prime}\right)$, which is a permutation of $\left(h_{0}, h_{1}, \cdots, h_{c}\right)$ put in the decreasing order.

Proof. See [3] Proposition 18.

Proposition 6. With the same notation as above, suppose that $J \in \operatorname{End}(A)$ is a strong Lefschetz element. Then any homogeneous basis of Ker $J$ can be extended uniquely to a homogeneous Jordan basis for $J$.

Proof. Let $\sum h_{i} q^{i}$ be the Hilbert series of $A$. Since $J$ is a strong Lefschetz element, we have

$$
\operatorname{dim}\left(\operatorname{Ker} J \cap A_{i}\right)= \begin{cases}0 & \text { if } h_{i} \leq h_{i+1}, \\ h_{i}-h_{i+1} & \text { if } h_{i}>h_{i+1}\end{cases}
$$


Let $s$ be the greatest integer such that $h_{s-1}<h_{s}$ and let

$$
m_{i}=h_{i}-h_{i-1} \text {, for } i=0,1,2, \cdots, s .
$$

Since $h_{i}=h_{c-i}$ for $0 \leq i \leq c$, we may rewrite the equation (8) as

$$
\operatorname{dim}\left(\operatorname{Ker} J \cap A_{c-i}\right)= \begin{cases}m_{i} & \text { for } i=0,1,2, \cdots, s, \\ 0 & \text { otherwise. }\end{cases}
$$

Now let $B$ be a homogeneous basis of $\operatorname{Ker} J$ given arbitrarily. We have to find a basis of $A$ containing $B$ on which $J$ is in the Jordan canonical form. Put $B_{c-i}=B \cap A_{c-i}$ for $i=0,1, \cdots, s$. Then $B_{c-i}$ is a basis of $\operatorname{Ker} J \cap A_{c-i}$. Since the restricted map $J^{c-2 i}: A_{i} \rightarrow A_{c-i}$ is bijective, there is a finite $\operatorname{set} B_{i} \subset A_{i}$ such that $\# B_{i}=m_{i}$ and such that $J^{c-2 i}\left(B_{i}\right)=B_{c-i}$. It is easy to show that the set

$$
\widetilde{B}:=\bigsqcup_{i=0}^{s}\left\{J^{j}\left(B_{i}\right) \mid j=0,1,2, \cdots, c-2 i\right\}
$$

is linearly independent and hence is a basis of $A$. It is easy to see that $\widetilde{B}$ is a desired basis. The uniqueness is obvious since the choice of the finite set $B_{i}$ is unique for $i=0,1, \cdots, s$.

The following result is proved in [9] and plays an important role in this paper.

Theorem 7. Let $A=A(n, k)$ be the same as defined in Section 1 . Then $A$ has the strong Lefschetz property and $x_{1}+x_{2}+\cdots+x_{k}$ is a strong Lefschetz element of $A$.

\section{3. $A(n, 2)$, the two fold tensor of $K^{n}$.}

If $k=2$, then the Gorenstein algebra $A=K\left[x_{1}, \cdots, x_{k}\right] /\left(x_{1}^{n}, \cdots, x_{k}^{n}\right)$ takes the form

$$
A=K\left[x_{1}, x_{2}\right] /\left(x_{1}^{n}, x_{2}^{n}\right) .
$$

Write $x, y$ for $x_{1}, x_{2}$. Recall that we identify $A=A(n, 2)$ as a vector subspace of $R=K[x, y]$ and $R=A \oplus\left(x^{n}, y^{n}\right)$. Denote by $\times: A \rightarrow \operatorname{End}(A)$ the regular representation of the Artinian algebra $A$. Put $J=\times(x+y)$. Since $J$ is a strong Lefschetz element, we may use Proposition 6 to construct a Jordan basis for $J$. First we would like to construct a homogeneous basis for $\operatorname{Ker} J$. 
The Hilbert series of $A$ is the following sequence.

\begin{tabular}{|l|c|c|c|c|c|c|c|c|c|c|}
\hline degree & 0 & 1 & 2 & $\cdots$ & $n-2$ & $n-1$ & $n$ & $\cdots$ & $2 n-3$ & $2 n-2$ \\
\hline $\operatorname{dim}$ & 1 & 2 & 3 & $\cdots$ & $n-1$ & $n$ & $n-1$ & $\cdots$ & 2 & 1 \\
\hline
\end{tabular}

Since $J$ is a strong Lefschetz element, $J: A_{i} \rightarrow A_{i+1}$ is either injective or surjective. Hence we have

$$
\operatorname{dim}\left(\operatorname{Ker} J \cap A_{i}\right)=\left\{\begin{array}{l}
0, \text { if } i<n-1, \\
1, \text { if } n-1 \leq i \leq 2 n-2 .
\end{array}\right.
$$

Since $\operatorname{dim}\left(\operatorname{Ker} J \cap A_{i}\right)$ is at most one, a homogeneous basis of $\operatorname{Ker} J$ is uniquely determined up to constant multiple. For $d=n-1$, $n, n+1, \cdots, 2 n-2$, put

$$
b_{d}=\sum_{j=0}^{d}(-1)^{j} x^{d-j} y^{j}, \text { and } \bar{b}_{d}=b_{d} \bmod \left(x^{n}, y^{n}\right) .
$$

Then since $b_{d}(x+y)=x^{d+1} \pm y^{d+1}$, we have $\bar{b}_{d} \in$ Ker $J$ for $d \geq n-1$. Thus we have

$$
\operatorname{Ker} J \cap A_{d}=\left\langle\bar{b}_{d}\right\rangle \text { for } d=n-1, n, \cdots, 2 n-2 .
$$

Because $J$ is a strong Lefschetz element, there is an element $a_{i} \in A_{i}$ for each $i \leq n-1$ such that $J^{2 n-2-2 i}\left(a_{i}\right)=\bar{b}_{2 n-2-i}$. Note that $J^{j}\left(a_{i}\right)$ are all symmetric if $i$ is even and are alternating if $i$ is odd. Now we have proved the following.

THEOREM 8. The set

$$
\bigsqcup_{i=0}^{n-1}\left\{J^{j}\left(a_{i}\right) \mid j=0,1,2, \cdots, 2 n-2-2 i\right\}
$$

is a homogeneous Jordan basis for $J \in \operatorname{End}(A)$. The basis element $J^{j}\left(a_{i}\right)$ is symmetric if $i$ is even and alternating if $i$ is odd. The conjugacy class of $J$ is given by

$$
\lambda(J)=(2 n-1,2 n-3, \cdots, 3,1) .
$$

Proof. The first part was treated more generally in Proposition 6. The second part follows immediately from the definition of $b_{2 n-2-i}$ and $a_{i}$. The third statement follows from Proposition 5. 
Now we consider the representation

$$
\Phi: G L(n) \rightarrow G L(A)
$$

as introduced in Section 2.2. (For the definition of $\Phi$ see (3) and (4).)

Recall that the special linear group $S L(2)$ has a unique irreducible module of dimension $i$ for each $i>0$. We denote it by $V(i-1)$. Fix $n>0$ and let

$$
\Psi: S L(2) \rightarrow G L(n)
$$

be the irreducible representation corresponding to the module $V(n-1)$. We may consider $A=A(n, 2)$ as an $S L(2)$-module via the composition

$$
S L(2) \stackrel{\Psi}{\rightarrow} G L(n) \stackrel{\Phi}{\rightarrow} G L(A) .
$$

Proposition 9. With the same notation above A decomposes into SL(2)modules as

$$
A \cong V(2 n-2) \oplus V(2 n-4) \oplus \cdots \oplus V(0) .
$$

Proof. Abbreviate $\rho=\Phi \circ \Psi$, so $\rho: S L(2) \rightarrow G L(A)$. The group homomorphism $\rho$ induces a Lie algebra homomorphism

$$
d \rho: \mathfrak{g l}(2) \rightarrow \mathfrak{g l}(A) .
$$

It is well known that the irreducible decomposition of $\rho$ is determined by that of $d \rho$. Now recall that the Lie algebra sl(2) is the vector space spanned by three elements $e, f, h$ with the bracket relations

$$
[e, f]=h,[h, e]=2 e,[h, f]=-2 f .
$$

It is easy to see that to decompose $A$ into irreducible $\mathfrak{s I}(2)$-modules is to decompose $d \rho(e)$ into Jordan blocks (cf. [9]). Notice that $d \Psi(e)$ is nilpotent and may be considered as a single Jordan block by conjugation since $\Psi$ is irreducible. Consequently $d \rho(e) \in \mathfrak{g l}(A)$ may be considered as the multiplication map $\times(x+y)$ by definition of $\rho$ and $d \rho$. Hence the assertion follows from Theorem 8.

Remark 10. The isomorphism in Proposition 9 is known as the ClebschGordan decomposition of the tensor product $V(n-1) \otimes V(n-1)$ as $\mathfrak{s})(2)$ modules. We may also regard it as a consequence of Pieri's formula as follows. Let $\rho_{\lambda}$ denote the irreducible representation of $G L(n)$ corresponding to a Young diagram $\lambda$ with $l(\lambda) \leq n$. Pieri's formula (e.g. [6, I 
(5.16)]) applied to the character proves that

$$
\rho_{\mu} \otimes \rho_{(r)} \cong \bigoplus_{\lambda} \rho_{\lambda}
$$

where the sum is taken over all partitions $\lambda$ such that $\lambda / \mu$ is a horizontal $r$ strip. When $n=2$ and $\mu=(r)$ and all $\rho_{\lambda}$ are restricted to $S L(2)$, it gives us the decompostion of Proposition 9 with $r$ replacing $n-1$.

With the same notation as above let $W=V(n-1) \otimes V(n-1)$. Let $W=W_{s} \oplus W_{a}$ be the decomposition of $W$ into the symmetric and alternating tensors respectively. (It is well known that the spaces $W_{s}$ and $W_{a}$ are irreducible $G L(n)$-modules, which we take for granted.) Via the representation (10) the spaces $W_{s}$ and $W_{a}$ are also $S L(2)$-modules. The following proposition shows how $W_{s}$ and $W_{a}$ decompose into irreducible $S L(2)$-modules.

Proposition 11. If $n$ is even, then

$$
W_{s} \cong \underbrace{V(2 n-2) \oplus V(2 n-6) \oplus \cdots \oplus V(2)}_{n / 2},
$$

and

$$
W_{a} \cong \underbrace{V(2 n-4) \oplus V(2 n-8) \oplus \cdots \oplus V(0)}_{n / 2} .
$$

If $n$ is odd, then

$$
W_{s} \cong \underbrace{V(2 n-2) \oplus V(2 n-6) \oplus \cdots \oplus V(0)}_{(n+1) / 2},
$$

and

$$
W_{a} \cong \underbrace{V(2 n-4) \oplus V(2 n-8) \oplus \cdots \oplus V(2)}_{(n-1) / 2} .
$$

Proof. Identify $W=A(n, 2)$. Then $W_{s}$ is the space spanned by the symmetric polynomials in $A$ and $W_{a}$ the alternating polynomials. Hence the assertion follows immediately from Theorem 8 and Proposition 9.

REMARK 12. Let $\rho=\Phi \circ \Psi$ be the composite

$$
S L(2) \stackrel{\Psi}{\rightarrow} G L(n) \stackrel{\Phi}{\rightarrow} G L(W)
$$


as defined in (11). Let $\rho=\rho_{s} \oplus \rho_{a}$ be the decomposition corresponding to $W=W_{s} \oplus W_{a}$. Let $h=\operatorname{diag}\left(q^{-1}, q\right) \in S L(2)$. The plethysm formula (e.g. [6, (I.8) Example 9)]) can be used to obtain the diagonal form for the matrices $\rho_{s}(h)$ and $\rho_{a}(h)$ and the Jordan decomposition for

$$
d \rho\left(\left(\begin{array}{ll}
0 & 1 \\
0 & 0
\end{array}\right)\right)=L
$$

As is conceived, it gives us the same decomposition as Proposition 11. In particular, it provides us with another proof for the fact that $x+y$ is a strong Lefschetz element in the Artinian algebra $R /\left(x^{n}, y^{n}\right)$.

\section{4. $A(2, k)$, the $k$-fold tensor of $K^{2}$.}

Throughout this section we fix $A=A(2, k)$. So $A$ is the subspace of the polynomial ring $K\left[x_{1}, \cdots, x_{k}\right]$ spanned by square-free monomials. At the same time $A$ is endowed with the algebra structure with the identification:

$$
A=K\left[x_{1}, \cdots, x_{k}\right] /\left(x_{1}^{2}, \cdots, x_{k}^{2}\right) .
$$

We put $L=\times\left(x_{1}+x_{2}+\cdots+x_{k}\right)$ and

$$
D=\frac{\partial}{\partial x_{1}}+\cdots+\frac{\partial}{\partial x_{k}} .
$$

We think of $L$ and $D$ as operating on the polynomial ring $R=$ $=K\left[x_{1}, \cdots, x_{k}\right]$. Recall that $R=A \oplus I$, where $I=\left(x_{1}^{2}, \cdots, x_{k}^{2}\right)$. We denote by $\left.D\right|_{A}$ the restricted map $D$ on $A$. Similarly by $\left.L\right|_{A}$ we denote the map $R / I \rightarrow R / I$ induced by $L$. Thus $\left.L\right|_{A},\left.D\right|_{A} \in \operatorname{End}(A)$. Let $H$ be the commutator $H=\left[\left.L\right|_{A},\left.D\right|_{A}\right]$. So $H \in \operatorname{End}(A)$.

Proposition 13. (a) $\left\langle\left. L\right|_{A},\left.D\right|_{A}, H\right\rangle$ is an $\mathfrak{s l}(2)$-triple.

(b) There exists a Jordan basis $B$ for $\left.L\right|_{A}$ such that $B \backslash \operatorname{Im}\left(\left.L\right|_{A}\right)$ is a basis of $\operatorname{Ker}\left(\left.D\right|_{A}\right)$.

Proof. (a) Let $J_{+}=\left(\begin{array}{ll}0 & 1 \\ 0 & 0\end{array}\right), J_{-}=\left(\begin{array}{ll}0 & 0 \\ 1 & 0\end{array}\right)$. Then the three elements $\left\langle J_{+}, J_{-},\left[J_{+}, J_{-}\right]\right\rangle$, where $\left[J_{+}, J_{-}\right]=\left(\begin{array}{cc}1 & 0 \\ 0 & -1\end{array}\right)$, is an $\mathfrak{s}(2)$-triple. This proves the case $k=1$. Let $E_{2}$ be the $2 \times 2$ identity matrix. Then one sees that, using square free monomials as a basis of $A$, the map $\left.L\right|_{A}$ is represented by 
the matrix

$$
\sum_{i=1}^{k} \underbrace{E_{2} \otimes \cdots \otimes E_{2}}_{i-1} \otimes J_{+} \otimes \underbrace{E_{2} \otimes \cdots \otimes E_{2}}_{k-i}
$$

and similarly $\left.D\right|_{A}$ by

$$
\sum_{i=1}^{k} \underbrace{E_{2} \otimes \cdots \otimes E_{2}}_{i-1} \otimes J_{-} \otimes \underbrace{E_{2} \otimes \cdots \otimes E_{2}}_{k-i} .
$$

We induct on $k$ to show that the three matrices $\left.L\right|_{A},\left.D\right|_{A}$, and $H:=\left[\left.L\right|_{A},\left.D\right|_{A}\right]$ satisfy the required relations $\left[H,\left.L\right|_{A}\right]=\left.2 L\right|_{A}$, and $\left[H,\left.D\right|_{A}\right]=-\left.2 D\right|_{A}$.

(b) See Humphreys ([4] pp. 31-34).

The following theorem enables us to construct a Jordan basis for $\left.L\right|_{A}$.

Theorem 14. For $i=0,1,2, \cdots,[k / 2]$, the vector space $(\operatorname{Ker} D) \cap A_{i}$ is spanned by the Specht polynomial of degree $i$. The Specht polynomials arising from the standard Young tableaux form a basis of $(\operatorname{Ker} D) \cap A$.

Proof of Theorem 14 is postponed to the end of Proposition 18.

LEMMA 15. $\operatorname{Ker} D=K\left[\left\{x_{i}-x_{j} \mid 1 \leq i, j \leq k\right\}\right]$.

Proof. Recall that $R=K\left[x_{1}, \cdots, x_{k}\right]$ and $\operatorname{Ker} D=\{f \in R \mid D f=0\}$. Since $\operatorname{Ker} D$ is a subalgebra of $R$, we have

$$
\operatorname{Ker} D \supset K\left[\left\{x_{i}-x_{j} \mid 1 \leq i, j \leq k\right\}\right] .
$$

The right hand side is isomorphic to the polynomial ring in $(k-1)$ variables. Noticing that $D$ is surjective of degree -1 , it is easily verified that they coincide by comparing the Hilbert series.

Lemma 16. Put $V=(\operatorname{Ker} D) \cap A$. Then

$$
\operatorname{dim} V_{i}=\operatorname{Max}\left\{\left(\begin{array}{c}
k \\
i
\end{array}\right)-\left(\begin{array}{c}
k \\
i-1
\end{array}\right), 0\right\},
$$

where $V_{i}=V \cap A_{i}$.

Proof. First note that the Hilbert series of $A$ is given by $\sum\left(\begin{array}{l}k \\ i\end{array}\right) q^{i}$. 
The graded vector space $A$ has the strong Lefschetz property with $\left.L\right|_{A}$ a strong Lefschetz element. By Proposition 13, the maps $\left.D\right|_{A}$ and $\left.L\right|_{A}$ are alike except that $\left.D\right|_{A}$ is a degree -1 map. Thus $\left.D\right|_{A}: A_{i} \rightarrow A_{i-1}$ is either injective or surjective. Thus the assertion follows.

Lemma 17. Let $T$ be a Young tableau with the shape $\lambda$. Suppose that $\lambda$ has size k. Let $\Delta_{T}$ be the Specht polynomial defined by $T$.

(1) $\Delta_{T}=1 \Longleftrightarrow \lambda$ has one row.

(2) $\Delta_{T} \in A$ and $\Delta_{T} \neq 1 \Longleftrightarrow \lambda$ has two rows.

(3) Suppose that $\Delta_{T} \in A$. Then the degree of $\Delta_{T}$ is equal to the second term of $\lambda$.

(4) $\Delta_{T} \in \operatorname{Ker}\left[\left.D\right|_{A}: A \rightarrow A\right] \cap A_{i}$ if $\lambda=(k-i, i)$.

Proof. (1), (2) and (3) are immediate from the definition of $\Delta_{T}$. (4) follows from Lemma 15.

We need one more proposition to prove Theorem 14 .

Proposition 18. Suppose that $\lambda=(k-i, i)$ is a Young diagram.

(a) The number of standard Young tableaux of shape $\lambda$ is $\left(\begin{array}{c}k \\ i\end{array}\right)-\left(\begin{array}{c}k \\ i-1\end{array}\right)$.

(b) The set of Specht polynomials defined by the standard Young tableaux of a fixed shape $\lambda$ is linearly independent.

Proof. (a) Suppose that $T$ is a standard Young tableau. If the box $k$ is removed from $T$ it is a Standard Young tableau of size $k-1$. Thus the induction works. (Details are left to the reader.) (b) Suppose that $T$ is standard. Then one notices easily that the head term of $\Delta_{T}$ in the reverse lexicographic monomial order is the product of monomials in the second row. Thus the proof is complete.

Proof of Theorem 14. Immediate by Lemmas 15, 16, 17 and Proposition 18.

Now our main theorem of this section is stated as follows.

Theorem 19. Let $J=\left.L\right|_{A}$ and $V_{d}=\left(\left.\operatorname{Ker} D\right|_{A}\right) \cap A_{d}$. Put $h=[k / 2]$, and $m_{i}=\left(\begin{array}{c}k \\ i\end{array}\right)-\left(\begin{array}{c}k \\ i-1\end{array}\right)$ for $i=0,1, \ldots, h$. 
(1) The conjugacy class $\lambda(J)$ of $J$ is given by

$\lambda(J)=\left\{\begin{array}{l}(\underbrace{k+1, \cdots, k+1}_{m_{0}}, \underbrace{k-1, \cdots, k-1}_{m_{1}}, \underbrace{k-3, \cdots, k-3}_{m_{2}}, \cdots, \underbrace{1, \cdots, 1}_{m_{h}}), \text { if } k \text { is even }, \\ (\underbrace{k+1, \cdots, k+1}_{m_{0}}, \underbrace{k-1, \cdots, k-1}_{m_{1}}, \underbrace{k-3, \cdots, k-3}_{m_{2}}, \cdots, \underbrace{2, \cdots, 2}_{m_{h}}), \text { if } k \text { is odd. }\end{array}\right.$

(2) For $0 \leq d \leq h$, and $0 \leq i \leq k-2 d$, the vector space

$$
J^{i}\left(V_{d}\right)
$$

is an irreducible $S_{k}$-module of isomorphism type $\lambda=(k-d, d)$.

(3) For any Specht polynomial $\Delta_{T} \in V_{d}$, the vector space

$$
\left\langle\Delta_{T}, J\left(\Delta_{T}\right), J^{2}\left(\Delta_{T}\right) \cdots, J^{k-2 d}\left(\Delta_{T}\right)\right\rangle
$$

is an irreducible $G L(2)$-module of isomorphism type $\lambda=(k-d, d)$.

(4) An irreducible decomposition of $A$ as $S_{k}$-modules is given by

$$
A=\bigoplus_{d=0}^{h}\left(\bigoplus_{i=0}^{k-2 d} J^{i}\left(V_{d}\right)\right) .
$$

In particular the irreducible $S_{k}$-module of type $\lambda=(k-d, d)$ occurs $(k+1-2 d)$ times, and the irreducible $G L(2)$-module of type $(k-d, d)$ occurs $m_{d}$ times.

Proof. (1) See Proposition 5. (2) In Lemma 17 and Proposition 18 we showed that the space $V_{d}$ is spanned by the Specht polynomials defined by the standard Young tableau of shape $(k-d, d)$. It is well known that this is irreducible. (Cf. [8].) Also $l^{i}\left(V_{d}\right)$ is isomorphic to $V_{d}$ unless it is trivial because $x_{1}+\cdots+x_{k}$ is $S_{k}$-invariant. (3) Let $\rho$ be the composition

$$
S L(2) \stackrel{\Psi}{\rightarrow} G L(2) \stackrel{\Phi}{\rightarrow} G L(A),
$$

where $\Psi$ is the natural injection and $\Phi$ is the tensor representation. To decompose $A$ into irreducible $G L(2)$-modules is the same as to decompose it as $S L(2)$-modules. This is obtained by decomposing the Lie algebra representation:

$$
d \rho: \mathfrak{g l}(2) \rightarrow \mathfrak{g l}(A) .
$$

Now the assertion follows from Lemma 13. (4) Clear from (1), (2) and (3). 
ExAmple 20. Let $k=4$. Let $l=\times\left(x_{1}+x_{2}+x_{3}+x_{4}\right) \in \operatorname{End}(A(2,4))$. We exhibit the Jordan basis of $\times l$. The Hilbert series of $A$ is $(1,4,6,4,1)$. The derived sequence is $(1,3,2)$.

1. The Specht polynomial of degree 0 is 1 .

2. The Specht polynomials of degree 1 (corresponding to the standard Young tableau with shape $\lambda=(3,1))$ are $a:=x_{1}-x_{2}, b:=x_{1}-x_{3}$ and $c:=x_{1}-x_{4}$.

3. The Specht polynomials of degree 2 (corresponding to the standard Young tableau with shape $\lambda=(2,2))$ are $f:=\left(x_{1}-x_{2}\right)\left(x_{3}-x_{4}\right)$ and $g:=\left(x_{1}-x_{3}\right)\left(x_{2}-x_{4}\right)$.

The bases for the irreducible decomposition of $A=A(2,4)$ as $G L(2)$ modules are:

1. $\left\langle 1, l, l^{2}, l^{3}, l^{4}\right\rangle$

3. $\left\langle a, l a, l^{2} a\right\rangle$ and $\left\langle b, l b, l^{2} b\right\rangle$ and $\left\langle c, l c, l^{2} c\right\rangle$

3. $\langle f\rangle$ and $\langle g\rangle$

We have $2^{4}=5 \times 1+3 \times 3+1 \times 2$. When $l^{i}$ is expanded, all terms which contain a square of a variable should be regarded zero. With this convention $l^{i}$ is equal to the $i$ th elementary symmetric polynomial multiplied by $i$ !.

\section{Application to the theory of Gorenstein rings.}

Put $A=R /\left(x_{1}^{2}, \cdots, x_{k}^{2}\right)$ and $l=x_{1}+x_{2}+\cdots+x_{k} \in A$. Using the notation of Proposition 13, we have $\times l=\left.L\right|_{A}$. Since we have obtained a Jordan basis for $\left.D\right|_{A}: A \rightarrow A$, it gives us a basis for $0: l$ as well as for $\operatorname{Ker}[D: A \rightarrow A]$. (cf. Proposition 13.) However, it does not necessarily a minimal ideal basis for $0: l$. In this section we would like to exhibit a minimal basis of the ideal 0: $l$. Denote by ( $)^{\star}: A \rightarrow A$ the "Hodge dual" of $A$. Namely, define

$$
M^{\star}=\left(x_{1} \cdots x_{k}\right) / M
$$

for a monomial $M \in A$. By linearity this is extended to define the dual map $A \rightarrow A$. The following lemma is easy to see, and proof is omitted.

LEMMA 21. Let $\lambda=(r, s)$ be a Young diagram, with $r+s=k$, and let $\Delta$ be a Specht polynomial of shape $\lambda$. Then for any integer $i, 1 \leq i \leq k$, 
(1) $\frac{\partial}{\partial x_{i}} \Delta$ is either 0 or a Specht polynomial of shape $(r+1, s-1)$.

(2) $\left(\frac{\partial}{\partial x_{i}} F\right)^{\star}=x_{i}\left(F^{\star}\right)$ for any $F \in A$.

(3) $\left(\left(\frac{\partial}{\partial x_{1}}+\cdots+\frac{\partial}{\partial x_{k}}\right) F\right)^{\star}=\left(x_{1}+\cdots+x_{k}\right)\left(F^{\star}\right)$ for any $F \in A$.

In the next theorem $\Delta_{T}$ denotes the Specht polynomial defined by the Young tableau of some shape $\lambda$.

THEOREM 22. In the polynomial ring $R$, put

$$
I=\left(x_{1}^{2}, \cdots, x_{k}^{2}\right):\left(x_{1}+\cdots+x_{k}\right) .
$$

Then the minimal number of generators of the ideal I is

$$
k+\left(\begin{array}{l}
k \\
h
\end{array}\right)-\left(\begin{array}{c}
k \\
h+1
\end{array}\right) .
$$

Here $h$ is such that $h=k / 2$ or $h=(k+1) / 2$ according as $k$ is even or odd.

If $k$ is even, then

$$
I=\left(x_{1}^{2}, \cdots, x_{k}^{2}\right)+\left\{\Delta_{T}^{\star} \mid \operatorname{shape}(T)=(h, h)\right\} R,
$$

and if $k$ is odd,

$$
I=\left(x_{1}^{2}, \cdots, x_{k}^{2}\right)+\left\{\Delta_{T}^{\star} \mid \operatorname{shape}(T)=(h, h-1)\right\} R .
$$

(If $k$ is even, it is the same if $\star$ is dropped.)

Proof. It is enough to prove the second part. Put $V=$ $=\operatorname{Ker}[D: A \rightarrow A]$, and let $V_{i}$ be the homogeneous part of degree $i$ so that we have the decomposition $V=\bigoplus V_{i}$. First notice that

$$
\frac{\partial}{\partial x_{1}} V_{i}+\cdots+\frac{\partial}{\partial x_{k}} V_{i}=V_{i-1}
$$

by Lemma 21 (1). This is equivalent to

$$
x_{1} V_{i}^{\star}+\cdots+x_{k} V_{i}^{\star}=V_{i-1}^{\star}
$$

by Lemma 21 (2). We have already proved that the vector space $V$ is spanned by the Specht polynomials. Hence by Lemma 21 (3), we have that $0: l$ is spanned by $A_{T}^{\star}$ for various $T$. The above equality (13) shows that $0: l$ is, as an ideal, generated by $V_{h}^{\star}$. 
Corollary 23. Let $A=K\left[x_{1}, \cdots, x_{k}\right] /\left(x_{1}^{2}, \cdots, x_{k}^{2}\right)$, and let l be the linear element $l=x_{1}+\cdots+x_{k}$ of $A$. Then the minimal number of generators of the ideal $(0: l)$ is

$$
\left(\begin{array}{l}
k \\
h
\end{array}\right)-\left(\begin{array}{c}
k \\
h+1
\end{array}\right) .
$$

Here $h$ is as in Theorem 22.

Proof. Immediate by Theorem 22.

Theorem 24. As before let $A=R /\left(x_{1}^{2}, \cdots, x_{k}^{2}\right)$, and let $y \in A$ be a general linear form of $A$. Then the Macaulay type of $A /(y)$ is the hth Catalan number $\frac{1}{h+1}\left(\begin{array}{c}2 h \\ h\end{array}\right)$. Here $h$ is as in Theorem 22. Equivalently if we put $I=\left(x_{1}^{2}, \cdots, x_{k}^{2}, Y\right)$, where $Y$ is a general linear form of the polynomial ring and if we write a minimal free resolution of $R / I$ as

$$
0 \rightarrow F_{k} \rightarrow F_{k-1} \rightarrow \cdots \rightarrow F_{1} \rightarrow R \rightarrow R / I \rightarrow 0,
$$

then we have rank $F_{k}=\frac{1}{h+1}\left(\begin{array}{c}2 h \\ h\end{array}\right)$.

Proof. The Macaulay type of $A /(y)$ is equal to the minimal number of generators of 0: $y$. It is well known that this is also equal to the last rank of the minimal free resolution of $0: y$. Now it suffices to notice that

$$
\left(\begin{array}{l}
k \\
h
\end{array}\right)-\left(\begin{array}{c}
k \\
h+1
\end{array}\right)=\frac{1}{h+1}\left(\begin{array}{c}
2 h \\
h
\end{array}\right),
$$

where $h=k / 2$ or $(k+1) / 2$ as in Theorem 22 .

\section{The Hilbert series of the ring of invariants of $A(n, k)$.}

In this section we let $A=K\left[x_{1}, \cdots, x_{k}\right] /\left(x_{1}^{n}, \cdots, x_{k}^{n}\right)$ where $n$ and $k$ are arbitrary positive integers. Let $G:=S_{k}$ act on $A$ by permutation of the variables. In the next theorem we would like to exhibit the ring of invariants $A^{G}$ and the Hilbert series of $A^{G}$.

THEOREM 25.

$$
A^{G}=K\left[e_{1}, \cdots, e_{k}\right] /\left(p_{n}, p_{n+1}, \cdots, p_{n+k-1}\right) .
$$


Here $e_{d}$ is the elementary symmetric polynomial of degree $d$ and $p_{d}$ is the power sum $p_{d}=x_{1}^{d}+\cdots+x_{k}^{d}$. Hence the Hilbert series of $A^{G}$ is:

$$
h_{A^{G}}(q)=\frac{\left(1-q^{n}\right)\left(1-q^{n+1}\right) \cdots\left(1-q^{n+k-1}\right)}{\left(1-q^{1}\right)\left(1-q^{2}\right) \cdots\left(1-q^{k}\right)}
$$

Proof. Consider the exact sequence

$$
0 \rightarrow\left(x_{1}^{n}, \cdots, x_{k}^{n}\right) \rightarrow R \rightarrow A \rightarrow 0 .
$$

Since $\operatorname{ch} K=0$, we have the exact sequence

$$
0 \rightarrow\left(x_{1}^{n}, \cdots, x_{k}^{n}\right)^{G} \rightarrow R^{G} \rightarrow A^{G} \rightarrow 0 .
$$

Note that $R^{G}=K\left[e_{1}, \cdots, e_{k}\right]$. The socle degree of $A$ is $n k-k$, and $A_{n k-k}=\left\langle\left(e_{k}\right)^{n-1}\right\rangle$. Since $e_{k}^{n-1}$ is fixed under the action of $G$, this shows that the maximum degree of elements of $A^{G}$ is $n k-k$.

Put $A^{\prime}=R^{G} /\left(p_{n}, p_{n+1}, \cdots, p_{n+k-1}\right)$. Obviously we have a natural surjection:

$$
A^{\prime} \rightarrow A^{G} \rightarrow 0
$$

which we would like to prove to be an isomorphism. First note that the rational series in the statement of this theorem is the Hilbert series of $A^{\prime}$. (This can be obtained using the fact $R^{G}=K\left[e_{1}, e_{2}, \cdots, e_{k}\right]$.) This shows that $A^{\prime}$ and $A^{G}$ have the same socle degree, which is equal to $n k-k$. Since $A^{\prime}$ is an Artinian Gorenstein ring, the one dimensional vector space of the maximum degree is the unique minimal ideal of the ring $A^{\prime}$. This shows that the map (14) cannot have a non-trivial kernel. This completes the proof.

REMARK 26. 1. Since

$$
\lim _{q \rightarrow 1} \frac{\left(1-q^{n}\right)\left(1-q^{n+1}\right) \cdots\left(1-q^{n+k-1}\right)}{\left(1-q^{1}\right)\left(1-q^{2}\right) \cdots\left(1-q^{k}\right)}=\left(\begin{array}{c}
n+k-1 \\
k
\end{array}\right)
$$

we have $\operatorname{dim} A^{G}=h_{A^{G}}(1)=\left(\begin{array}{c}n+k-1 \\ k\end{array}\right)$. This is expected since $A^{G}$ is the irreducible $G L(n)$-module corresponding to the trivial $\lambda$, which is the symmetric tensor space.

2. One may conceive that the Hilbert series of $Y^{\lambda}(A)$ where $Y^{\lambda}$ is a Young symmetrizer corresponding to $\lambda=\left(k_{1}, \cdots, k_{r}\right) \vdash k$ should be obtained as a $q$-analog of the dimension formula of the irreducible $G L(n)$ module in the decomposition of $\left(K^{n}\right)^{\otimes k}$. This can be proved using the $q$ - 
dimension formula ([5] Proposition 10.10). We indicate an outline of proof in the next proposition.

Recall that we have the isomorphism $A(n, k) \cong\left(K^{n}\right)^{\otimes k}$ as vector spaces. The symmetric group $S_{k}$ acts on $A(n, k)$ as permutations of variables. Also the general linear group $G L(n)$ acts on $A(n, k)$ as the tensor representation. Recall that $Y^{\lambda}(A(n, k))=S_{k} \cdot Y^{T}(A(n, k))$, where the map $Y^{T}: A(n, k) \rightarrow A(n, k)$ is a Young symmetrizer with shape $\lambda \vdash k$ with at most $n$ parts. It is well known that $Y^{\lambda}(A(n, k))$ is an irreducible $\left(G L(n) \times S_{k}\right)$-module and every irreducible $G L(n)$-module is obtained as an irreducible component of $Y^{\lambda}(A(n, k)$ ) for some $\lambda$. (For details see, e.g., [2] pp. 336-339.)

Proposition 27. For $\lambda=\left(k_{1}, \ldots, k_{r}\right) \vdash k$ with $r \leq n$, the Hilbert series of the module $Y^{\lambda}(A(n, k))$ as a graded subspace of $A(n, k)$ is

$$
h_{Y^{\lambda}(A(n, k))}(q)=q^{n(\lambda)} \prod_{1 \leq i<j \leq n} \frac{\left[k_{i}-k_{j}+j-i\right]}{[j-i]} .
$$

Here $n(\lambda):=\sum_{j \geq 1}(j-1) k_{j},[a]$ denotes $\frac{1-q^{a}}{1-q}$ for any positive integer $a$, and $k_{j}=0$ for $j>r$.

Before we give a proof we review the $q$-dimension formula $[5, \S 10.9$, $\S 10.10]$ for $G L(n)$. Let $W$ be a finite dimensional irreducible $G L(n)$-module with highest weight $\lambda$, and $W=\bigoplus_{\mu} W_{\mu}$ its weight space decomposition. The $q$-dimension of $W$ is defined by

$$
\operatorname{dim}_{q} W=\sum_{\mu \text { : weight of } W}\left(\operatorname{dim} W_{\mu}\right) q^{\langle\lambda-\mu, \delta\rangle},
$$

where $\langle\cdot, \cdot\rangle$ denotes the standard inner product on $\mathbb{R}^{n}$, and $\delta$ is "the half sum of the positive roots" defined by $(n-1, n-3, \ldots,-n+1) / 2 \in \mathbb{R}^{n}$. Note that the exponent $\langle\lambda-\mu, \delta\rangle$ is the number of the simple roots occurring in $\lambda-\mu$, since $\langle\alpha, \delta\rangle=1$ for any simple root $\alpha$.

The $q$-dimension formula [5, Proposition 10.10] says

$$
\operatorname{dim}_{q} W=\prod_{1 \leq i<j \leq n} \frac{\left[k_{i}-k_{j}+j-i\right]}{[j-i]},
$$

where $W$ is a finite dimensional irreducible $G L(n)$-module with highest weight $\lambda=\left(k_{1}, \ldots, k_{n}\right)$. 
Proof of Proposition 27. - Let $G L(n)$ act on the $n$-dimensional $K$-vector space $K[x] /\left(x^{n}\right)$ through the vector representation with the basis $1, x, x^{2}, \ldots, x^{n-1}$. Then $x^{j-1}$ is a weight vector with weight $\varepsilon_{j}=(0, \ldots, 1, \ldots, 0)$ (only $j$ th entry is 1 ). Therefore the weight of a weight vector decreases by a single simple root (like $\varepsilon_{j}-\varepsilon_{j+1}$ ), as its degree increases by one. This principle holds also for $A(n, k)$, since $A(n, k)=K\left[x_{1}, \ldots, x_{k}\right] /\left(x_{1}^{n}, \ldots, x_{k}^{n}\right)$ is isomorphic to the tensor product $K\left[x_{1}\right] /\left(x_{1}^{n}\right) \otimes_{K} \cdots \otimes_{K} K\left[x_{k}\right] /\left(x_{k}^{n}\right)$ as $G L(n)$-modules. In particular, the homogeneous polynomial with the least degree in $Y^{\lambda}(A(n, k)) \subset A(n, k)$ is the highest weight vector.

It follows from the principle above and the note after (15) that the Hilbert series and the $q$-dimension of $Y^{\lambda}(A(n, k))$ differ only by a power of $q$. Notice that the $q$-dimension $\operatorname{dim}_{q} Y^{\lambda}(A(n, k))$ starts with $q^{0}$ followed by higher terms, while the Hilbert series $h_{Y^{\lambda}(A(n, k))}(q)$ starts with $q^{d}$, where $d$ is the degree of the monomial of the highest weight vector. Therefore it suffices to show that the degree of a monomial with weight $\lambda=\left(k_{1}, \ldots, k_{r}\right) \vdash k(r \leq n)$ is equal to $n(\lambda)$.

Since the weight of 1 is $\varepsilon_{1}=(1,0, \ldots, 0)$ in the $G L(n)$-module $K[x] /\left(x^{n}\right)$, the weight of 1 in $A(n, k) \simeq\left(K[x] /\left(x^{n}\right)\right)^{\otimes k}$ is $k \varepsilon_{1}=(k, 0, \ldots, 0)$. The degree of a monomial with weight $\lambda$ is

$$
\begin{aligned}
\left\langle k_{1}-\lambda, \delta\right\rangle & =\left\langle\left(k-k_{1},-k_{2},-k_{3}, \ldots,-k_{r}\right), \delta\right\rangle \\
& =k_{2}+2 k_{3}+\cdots+(r-1) k_{r} .
\end{aligned}
$$

We thus have proved the assertion.

\section{The multiplicative group of the Artinian algebras.}

We need the following proposition from commutative algebra. Very important to us is the corollary that follows, which says that the generic form of degree $d$ has the largest rank among all elements of order $d$. The proof for the case where $M$ is principal is given in [10, Proposition A in Appendix]. Since the proof goes verbatim, we omit the proof.

Proposition 28. Let $(R, \mathfrak{m})$ be an Artinian local ring with residue field $K$ and $M$ a finite $R$-module. Let $m_{1}, \cdots, m_{s}$ be arbitrary elements of $\mathfrak{m}$, and let $l=x_{1} m_{1}+x_{2} m_{2}+\cdots+x_{s} m_{s}$ with $x_{i} \in R$. Let $X_{1}, \cdots, X_{s}$ be indeterminates over $R$, let $Q=R\left[X_{1}, \cdots, X_{s}\right]$ be the polynomial ring and let

$$
\widetilde{M}=M \otimes_{R} Q / Y\left(M \otimes_{R} Q\right) .
$$


where $Y=X_{1} m_{1}+\cdots+X_{s} m_{s}$. Let $U$ be the set of non-zero-divisors of $Q$ and let a be the ideal $\left(X_{1}-x_{1}, \cdots, X_{s}-x_{s}\right) Q$. Then we have

$$
\operatorname{length}_{Q_{U}}\left(\widetilde{M}_{U}\right) \leq \operatorname{length}_{Q / \mathfrak{a}}\left(\widetilde{M} \otimes_{Q} Q / \mathfrak{a}\right) .
$$

Furthermore, there exists a radical ideal $\mathfrak{b} \subset K\left[X_{1}, \ldots, X_{s}\right]$ such that the inequality (17) is the equality if and only if the ideal $\left(X_{1}-\bar{x}_{1}, \ldots, X_{s}-\bar{x}_{s}\right)$ does not contain $\mathfrak{b}$. ( $\bar{x}_{i}$ denotes the image of $x_{i}$ in $\left.K.\right)$

COROLlary 29. Suppose that $A=\bigoplus_{i=0}^{c} A_{i}$ is a standard graded $K$-algebra, where $K$ is an infinite field. Let $\stackrel{i=0}{M}=\bigoplus_{i=a}^{b} M_{i}$ be a finite graded $A$ module. Let $\mathrm{m}$ be the maximal ideal of $A$. Let $d$ be any positive integer. Then the minimal value

$$
\operatorname{Min}\{\operatorname{dim} M / y M\},
$$

where $y$ ranges over $\mathrm{m}^{d}$, is attained by a homogeneous element $y$ of degree d. If $M$ has the strong Lefschetz property, and if $z \in A_{1}$ is a strong Lefschetz element for $M$, then we have

$$
\operatorname{dim} M / z^{d} M=\operatorname{Min}\left\{\operatorname{dim} M / y M \mid y \in \mathfrak{m}^{d}\right\},
$$

or equivalently, the rank of the linear map $\times z^{d}: M \rightarrow M$ is greater than or equal to the rank of $\times y: M \rightarrow M$ for any $y \in \mathfrak{m}^{d}$.

Proof. Since $\mathfrak{m}^{d}=\bigoplus_{j \geq d} A_{j}$ and since $\mathfrak{m}^{d} / \mathfrak{m}^{d+1} \cong A_{d}$, any representative of a $K$-basis of $\mathfrak{m}^{d} / \mathfrak{m}^{d+1}$ is a minimal generating set of the ideal $\mathfrak{m}^{d}$ and vice versa. Thus the first part is a direct consequence of Proposition 28. (We apply the proposition by letting $m_{1}, \cdots, m_{s}$ be a set of minimal generating set of $\mathfrak{m}^{d}$.) To see the second part suppose that $y \in \mathfrak{m}^{d}$ is any homogeneous element. The linear map $\times y: M \rightarrow M$ decomposes into the sum of piece-wise linear maps $\times y: M_{i} \rightarrow M_{i+d}$. Hence the rank of the linear map $\times y: M \rightarrow M$ does not exceed

$$
\sum_{i=a}^{b-d} \operatorname{Min}\left\{\operatorname{dim} M_{i}, \operatorname{dim} M_{i+d}\right\} .
$$

(Here we have set $M_{b+i}=0$ for $i>0$.) Now if $z$ is a strong Lefschetz element and if $y=z^{d}$, then the rank of $\times y: M \rightarrow M$ is equal to the integer given in (18) above. In fact the linear map $\times z^{d}: M_{i} \rightarrow M_{i+d}$ is either injective or surjective as is easily deduced from the definition. Thus we have shown that the rank of the linear map $\times z^{d}: M \rightarrow M$ is greater than or equal 
to the rank of $y^{\prime}: M \rightarrow M$ for any $y^{\prime} \in \mathfrak{m}^{d}$. This is equivalent to the second assertion of the corollary.

Let $A=\bigoplus_{i=0}^{c} A_{i}$ be an Artinian standard graded $K$-algebra. We denote by $A^{*}$ the multiplicative group of $A$. Obviously it is an Abelian algebraic group with the underlying algebraic set

$$
\left\{\left(a_{0}, a_{1}, \cdots, a_{c}\right) \in A=\bigoplus A_{i} \mid a_{i} \in A_{i}, a_{0} \neq 0\right\} .
$$

We may regard it as an algebraic subgroup of $G L(A)$.

Example 30. Let $A=K[x] /\left(x^{n}\right)$. Then $f \in A^{*}$ induces an automorphism $\times f: A \rightarrow A$. If we write

$$
f=a_{0}+a_{1} x+a_{2} x^{2}+\cdots+a_{n-1} x^{n-1},
$$

then $\times f$ is represented by the matrix

$$
\left(\begin{array}{cccccc}
a_{0} & a_{1} & a_{2} & \cdots & a_{n-2} & a_{n-1} \\
& a_{0} & a_{1} & \cdots & \cdots & a_{n-2} \\
& & a_{0} & \cdots & \cdots & a_{n-3} \\
& & & \ddots & \ddots & \vdots \\
& & & & \ddots & \vdots \\
& & & & & a_{0}
\end{array}\right) .
$$

This way we regard the group $A^{*}$ as the algebraic subgroup of $G L(n)$.

Let $V$ be an $n$-dimensional vector space and let

$$
\Phi: G L(V) \rightarrow G L\left(V^{\otimes k}\right)
$$

be the tensor representation. Now let $V=K[x] /\left(x^{n}\right)$ be as in Example 30 above and let $A=A(n, k)=K\left[x_{1}, \cdots, x_{k}\right] /\left(x_{1}^{n}, \cdots, x_{k}^{n}\right)$. Define the map

$$
\Phi^{\prime}: V^{*} \rightarrow A^{*}
$$

by

$$
f(x) \mapsto f\left(x_{1}\right) f\left(x_{2}\right) \cdots f\left(x_{k}\right) .
$$

Then it is easy to see that $\Phi^{\prime}$ is a group homomorphism. As described above we may identify $V^{*}$ as a subgroup of $G L(V)$ and $A^{*}$ a subgroup of $G L\left(V^{\otimes k}\right)$. Note that $\Phi^{\prime}$ is nothing but the restriction of $\Phi$. In other words we have the commutative diagram: 


$$
\begin{array}{ccc}
G L(V) & \stackrel{\Phi}{\rightarrow} & G L(A) \\
\uparrow & & \uparrow \\
V^{*} & \stackrel{\Phi^{\prime}}{\longrightarrow} & A^{*}
\end{array}
$$

where the vertical maps are natural inclusions.

By $J(a, n)$ we denote the $n \times n$ matrix

$$
J(a, n)=\left[\begin{array}{cccc}
a & 1 & & 0 \\
& a & \ddots & \\
& & \ddots & 1 \\
0 & & & a
\end{array}\right]
$$

It is an elementary fact that if a matrix $M$ has a single eigenvalue $a$, then $M$ decomposes into a direct sum of such blocks as follows:

$$
M=\left[\begin{array}{cccc}
J\left(a, n_{1}\right) & & & 0 \\
& J\left(a, n_{2}\right) & & \\
0 & & \ddots & \\
& & J\left(a, n_{r}\right)
\end{array}\right] .
$$

This is known as the Jordan canonical form of $M$. We denote this matrix by

$$
J\left(a, n_{1}\right) \oplus \cdots \oplus J\left(a, n_{r}\right) .
$$
Definition 31. Let $V=\sum_{i=a}^{b} V_{i}$ be a graded $K$-vector space with the
Hilbert series

$$
h(q):=h_{V}(q)=\sum_{i=a}^{b} h_{i} q^{i}
$$

where we assume that $V_{a} \neq 0$ and $V_{b} \neq 0$. Suppose that $h(q)$ is symmetric and unimodal. Then the dual Hilbert series of $V$, or of $h(q)$, is defined to be the descending sequence of positive integers

$$
u_{1}, u_{2}, \cdots, u_{s}
$$

which satisfy

$$
h(q)=\frac{1}{1-q} \sum_{i=1}^{r}\left(q^{\left(a+b+1-u_{i}\right) / 2}-q^{\left(a+b+1+u_{i}\right) / 2}\right) .
$$


REMARK 32. With the same notation as above, the Hilbert series $h(q)$ of $V$ gives a partition of the positive integer $\operatorname{dim} V$. In fact $\operatorname{dim} V=$ $=h_{a}+h_{a+1}+\cdots+h_{b}$. (If one prefers, the summands may be arranged in the increasing or decreasing order.) The dual Hilbert series of $V$ is nothing but the dual partition of the Hilbert series. For example if $h(q)=q^{a}+3 q^{a+1}+6 q^{a+2}+7 q^{a+3}+6 q^{a+4}+3 q^{a+5}+q^{a+6}$, then the dual Hilbert series of $V$ is $7,5,5,3,3,3,1$, independent of the shift by $a$.

Now we may state the main theorems of this section.

THEOREM 33. Let $\Phi: G L(n) \rightarrow G L\left(\left(K^{n}\right)^{\otimes k}\right)$ be the tensor representation of the general linear group $G L(n)$. Let $M=J(a, n)$. Then $a^{k} i s$ the unique eigenvalue of $\Phi(M)$ and the Jordan canonical form of $\Phi(M)$ is given by

$$
J\left(a^{k}, u_{1}\right) \oplus \cdots \oplus J\left(a^{k}, u_{r}\right),
$$

where $u_{1}, \cdots, u_{r}$ is the dual Hilbert series of the polynomial $h(q)=$ $=\left(1+q+\cdots+q^{n-1}\right)^{k}$.

Proof. Put $A=A(n, k)=K\left[x_{1}, \cdots, x_{k}\right] /\left(x_{1}^{n}, \cdots, x_{k}^{n}\right)$. Then, since $\left(1+\cdots+q^{n-1}\right)^{k}$ is the Hilbert series of $A$, it suffices to prove that (1) $a^{k}$ is the unique eigenvalue of $\Phi(M)$ and (2) $\Phi(M)-a^{k}$ has the same Jordan canonical form as that of a strong Lefschetz element of $A$. (See Proposition 6.) Put $V=K[x] /\left(x^{n}\right)$. We have the commutative diagram (19) as shown above. With the natural embedding $V^{*} \rightarrow G L(n)$ the element $a+x$ corresponds to the matrix $J(a, n)$. Thus $\Phi^{\prime}(a+\bar{x})=\Phi(M)$. Now put $l^{\prime}=\Phi^{\prime}(a+\bar{x})-a^{k}$. Then we have

$l^{\prime}=\left(a+\bar{x}_{1}\right) \cdots\left(a+\bar{x}_{k}\right)-a^{k}=a^{k-1}\left(\bar{x}_{1}+\cdots+\bar{x}_{k}\right)+$ polynomial in $\bar{x}_{i}$ of degree $\geq 2$.

Put $l=\bar{x}_{1}+\cdots+\bar{x}_{k}$. Then we have

$$
\left(\frac{1}{a^{k-1}} l^{\prime}\right)^{i} \equiv l^{i} \bmod \mathfrak{m}^{i+1}, i=1,2, \cdots .
$$

Thus by Proposition 28 we have that the rank of $\left(\times l^{i}\right)$ is equal to the rank of $\left(\times l^{i}\right)$ for all $i \geq 1$. This shows that $\Phi^{\prime}(a-\bar{x})-a^{k}$ and $l$ have the same Jordan canonical form. Now the proof is complete.

THEOREM 34. Let $\lambda \vdash k$ with at most $n$ parts and let $W^{\lambda}$ be an irreducible $G L(n)$-module corresponding to $\lambda$. Denote by $\phi^{\lambda}: G L(n) \rightarrow G L\left(W^{\lambda}\right)$ the corresponding irreducible representation of $G L(n)$. Put

$$
A(n, k)=K\left[x_{1}, \cdots, x_{k}\right] /\left(x_{1}^{n}, \cdots, x_{k}^{n}\right) .
$$


Suppose that the sequence

$$
(\underbrace{f_{1}, \cdots, f_{1}}_{m_{1}}, \underbrace{f_{2}, \cdots, f_{2}}_{m_{2}}, \cdots, \underbrace{f_{s}, \cdots, f_{s}}_{m_{s}})
$$

is the dual Hilbert series of $Y^{\lambda}(A(k, n))$. Then the Jordan canonical form of $\phi^{\lambda}(J(a, n))$ is given by

$\underbrace{J\left(a^{k}, f_{1}\right) \oplus \cdots \oplus J\left(a^{k}, f_{1}\right)}_{m_{1} / m} \oplus \underbrace{J\left(a^{k}, f_{2}\right) \oplus \cdots \oplus J\left(a^{k}, f_{2}\right)}_{m_{2} / m} \oplus \cdots \oplus \underbrace{J\left(a^{k}, f_{s}\right) \oplus \cdots \oplus J\left(a^{k}, f_{s}\right)}_{m_{s} / m}$,

where $m$ is the number of the standard Young tableaux of shape $\lambda$.

Before proving this theorem, we collect some basic facts in a lemma.

Lemma 35. Put $A=A(n, k)=K\left[x_{1}, \cdots, x_{k}\right] /\left(x_{1}^{n}, \cdots, x_{k}^{n}\right)$. Let $\lambda \vdash k$ and let $T$ be a standard Young tableau with shape $\lambda$. We identify $A$ with $\left(K^{n}\right)^{\otimes k}$. Then

(1) $Y^{\lambda}(A) \subset A$ is an irreducible $\left(G L(n) \times S_{k}\right)$-module.

(2) The number of times that the irreducible $G L(n)$-module $W^{\lambda}$ occurs in $Y^{\lambda}(A)$ is equal to the dimension of the irreducible $S_{k}$-module that occurs in $Y^{\lambda}(A)$.

(3) Suppose that $W \subset Y^{\lambda}(A)$ is an irreducible $G L(n)$-module. Let $m$ be the dimension of any irreducible $S_{k}$-module that occurs in $Y^{\lambda}(A)$. Then $h_{Y^{\lambda}(A)}(q)=m h_{W}(q)$.

(4) Let $l=\bar{x}_{1}+\cdots+\bar{x}_{k}$. Then $l$ is a strong Lefschetz element for $Y^{\lambda}(A)$. Hence the endomorphism $\times l \in \operatorname{End}\left(Y^{\lambda}(A)\right)$ decomposes as

$$
J\left(0, u_{1}\right) \oplus \cdots \oplus J\left(0, u_{r}\right),
$$

where $u_{1}, \cdots, u_{r}$ is the dual Hilbert series of $Y^{\lambda}(A)$.

Proof. (1) and (2) are immediate from the Schur-Weyl duality. Let $W \subset Y^{\lambda}(A)$ be an irreducible $G L(n)$-module and let $T_{1}, \cdots, T_{m}$ be all the standard Young tableaux with shape $\lambda$. Then we have

$$
Y^{\lambda}(A)=S_{k} \cdot W=Y^{T_{1}} W \oplus \cdots \oplus Y^{T_{m}} W .
$$

Thus (3) follows. We prove (4). Write $L$ for the linear map $\times l \in \operatorname{End}(A)$. Let $D \in \operatorname{End}(A)$ be the degree -1 map such that $D, L,[D, L]$ is an $\mathfrak{s l}(2)$-triple. We note that $D$ is compatible with the action of $S_{k}$ as well as $L$. Let $Z$ be any homogeneous basis for $0: l$. Then the set $\bigcup_{i>0} D^{i}(Z)$ is the Jordan basis for $L$, and the subset $Y^{\lambda}(A) \cap\left(\bigcup_{i \geq 0} D^{i}(Z)\right)$ is a Jordan basis of $\left.L\right|_{Y^{\lambda}(A)}$. This shows
(4). (cf. Proposition 7.) 
Proof of Theorem 34. - We use the notation of the lemma above. It is well known that $W^{\lambda}$ appears as a submodule of $\left(K^{n}\right)^{\otimes k}$. First recall that the dimension of an irreducible $S_{k}$-module in $Y^{\lambda}(A)$ is equal to the number of the standard Young tableaux of shape $\lambda$. To prove the assertion of the theorem it suffices to show that (1) $\Phi^{\prime}(a+x)$ restricted on the submodule $Y^{\lambda}(A)$ has the unique eigenvalue $a^{k}$ and (2) $l^{\prime}:=\Phi^{\prime}(a+x)-a^{k}$ decomposes into Jordan blocks in the same way as the multiplication $\times l \in \operatorname{End}\left(Y^{\lambda}(A)\right)$ decomposes into Jordan blocks. (It should be noticed beforehand that $l Y^{\lambda}(A) \subset Y^{\lambda}(A)$ and $l^{\prime} W^{\lambda} \subset W^{\lambda}$, which is easy to see.) By (4) of the lemma above, $l=\bar{x}_{1}+\cdots+\bar{x}_{k}$ is a strong Lefschetz element for $Y^{\lambda}(A)$. Hence the proof is complete.

COROLlaRY 36. The graded vector space $Y^{\lambda}(A)$ has a unimodal symmetric Hilbert series for any $\lambda \vdash k$.

Proof. The assertion is an immediate consequence of the fact that $l \in A$ is a strong Lefschetz element for $Y^{\lambda}(A)$. (cf. [5, Exercise 10.12].)

\section{REFERENCES}

[1] W. Fulton, Young Tableaux, London Mathematical Society Student Text 35, Cambridge University Press, 1997.

[2] R. Goodman - N. R. Wallach, Representations and Invariants of the Classical Groups, Cambridge University Press, 1998.

[3] T. Harima - J. Watanabe, The finite free extension of an Artinian K-algebra with the strong Lefschetz property, Rend. Sem. Mat. Univ. Padova, 110 (2003), pp. 119-146.

[4] J. E. Humphreys, Introduction to Lie Algebras and Representation Theory, Springer-Verlag, 1972.

[5] V. G. KAC, Infinite-dimensional Lie algebras, third ed., Cambridge University Press, Cambridge, 1990.

[6] I. G. Macdonald, Symmetric functions and Hall Polynomials, 2nd ed., Oxford Science Publications, London, 1995.

[7] T. Maeno, Lefschetz property, Schur-Weyl duality and a q-deformation of Specht polynomial, Comm. Algebra, 35 (2007), pp. 1307-1321.

[8] T. TERASOMA - H. -F. Yamada, Higher Specht polynomials for the symmetric group, Proc. Japan Acad., 69 (1993), pp. 41-44.

[9] J. WatAnABE, The Dilworth number of Artinian rings and finite posets with rank function, Adv. Stud. Pure Math., 11 (1987), pp. 303-312.

[10] J. WatanaBe, m-full ideals, Nagoya Math. J., 106 (1987), pp. 101-111.

[11] H. WEYL, Classical groups, their invariants and representations, 2nd Edition, Princeton, 1946.

Manoscritto pervenuto in redazione il 20 giugno 2007. 
